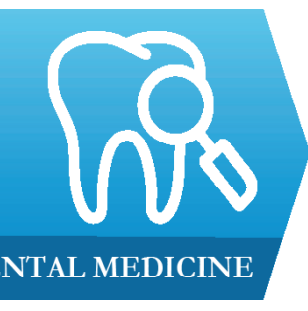

1) Department of Oral and Maxillofacial Surgery, Manipal College of Dental Sciences, Manipal Academy of Higher Education, Manipal, India

2) Department of Oral Medicine and Radiology, Manipal College of Dental Sciences, Manipal Academy of Higher Education, Manipal, India
DOI: $10.15386 / \mathrm{mpr}-1594$

Manuscript received: 12.02 .2020

Received in revised form: 23.06 .2020

Accepted: 10.07.2020

Address for correspondence:

komal.smriti@manipal.edu

This work is licensed under a Creative Commons Attribution-NonCommercialNoDerivatives 4.0 International License

\title{
Temporalis space infection secondary to an undiagnosed intra-oral foreign object - a case report
}

Anupam Singh ${ }^{1}$, Sreea Roy ${ }^{1}$, G Srikanth ${ }^{1}$, Shruti Gunashekhar ${ }^{1}$, Komal Smriti ${ }^{2}$

\begin{abstract}
The penetration of foreign objects is one of the leading causes of maxillofacial infection following trauma. Failure to detect such objects at initial stages can lead to complications like abscess formation, cellulitis, or space infections. Detection is even more complicated if the patient presents to the maxillofacial center after a delay of days or weeks following trauma. Sole reliance on radiographs or CT can be inconclusive as most of these objects are radiolucent and can be difficult to detect even by the experienced radiologists. We report the case of a patient who had an unwitnessed trauma and presented to our center 7 days after the incident, with signs of buccal space infection. Failure to detect the embedded intra-oral wooden object at an earlier stage led to the propagation of infection to superficial temporal space. The management strategy and pitfalls associated with conventional imaging in detecting wooden object are discussed.
\end{abstract}

Keywords: masticatory space infection, temporalis space infection, intra-oral foreign object, maxillofacial radiology

\section{Introduction}

Foreign bodies incorporated deep in tissues of the facial region represent a diagnostic dilemma. These penetrating injuries can result from missile injuries, blast injuries or accidental fall [1]. Around one-fourth of the embedded foreign materials go unnoticed on initial examination in an unwitnessed trauma [2]. The foreign particles which frequently get inserted into deeper facial structures after trauma are glass particles, pieces of wood, and pebbles. These materials are detected only by chance after taking radiographs or later, when the patient presents with recurrent infection or non-healing traumatic wound with complications like pain, abscess formation, collection of pus, swelling, etc. Appropriate and timely management of wooden foreign bodies are considered essential because of their potential infectious complications [3]. Several cases of embedded wooden foreign object go undiagnosed and untreated, despite the availability of excellent higher imaging diagnostic modality like ultrasound and CT. Here, we present the case of buccal space infection, which progressed to superficial temporal space due to pitfalls in diagnosing embedded foreign wooden piece after unwitnessed trauma.

\section{Case Report}

An otherwise healthy 61-year-old farmer reported to our hospital with a complaint of swelling on the left side of his face for one week. History revealed that the patient had a fall from a tree about a week ago and was initially seen at a local health care center. Preliminary care was done in the form of debridement of the lip and intra-oral laceration along with suturing, and administration of tetanus booster vaccine. The patient was discharged and prescribed a course of antibiotics and analgesics. However, the next day, the patient developed mild 
swelling and difficulty in mouth opening, which raised the suspicion of an underlying fracture or a developing infection. Subsequently, because of lack of any advanced imaging modality like ultrasound or CT scan, the patient was referred to our center for further evaluation. But the patient refused to seek any further treatment immediately because of financial constraints. Patient reported to the Department of Oral \& Maxillofacial Surgery, Manipal College of Dental Sciences, Manipal seven days after the trauma.

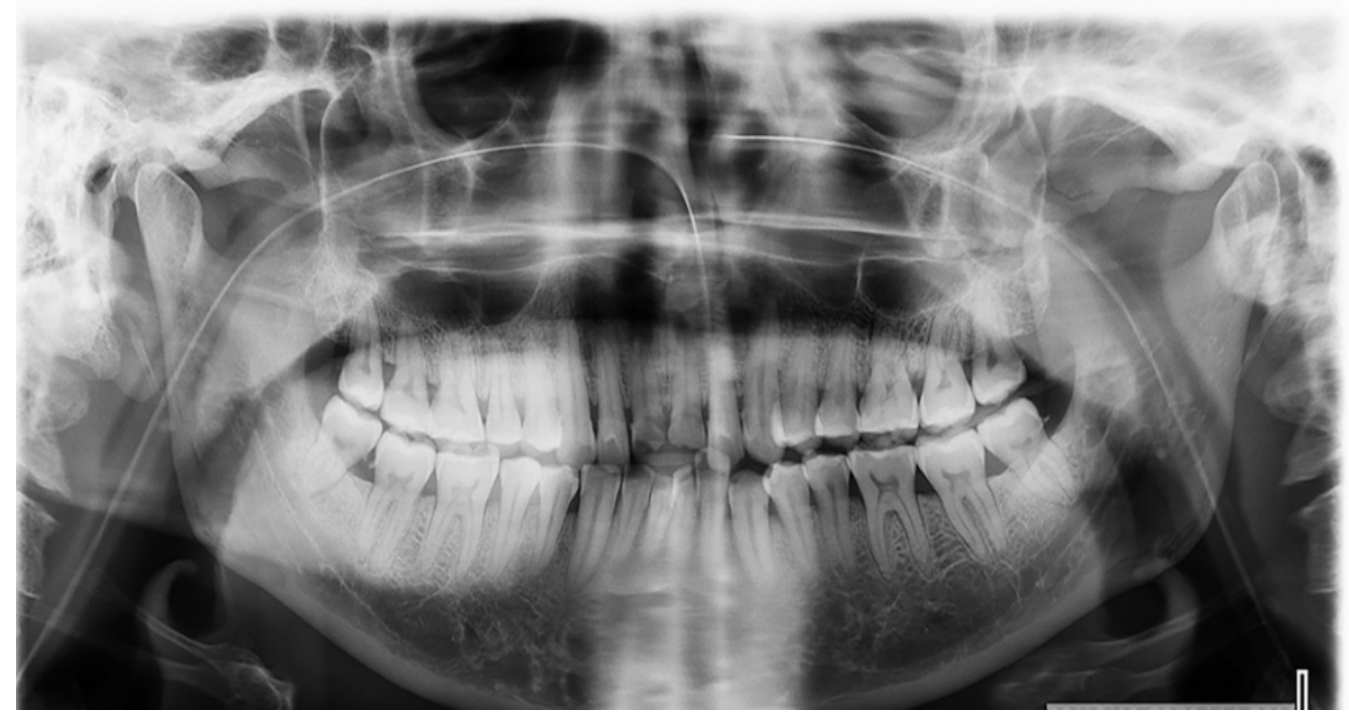

Figure 1. Orthopantomogram shows no evidence of fracture.

On examination, facial asymmetry was noted due to diffuse swelling of the left side of face, which was tender on palpation. No significant intra-oral finding was noted except for sutures on buccal mucosa and lip, however, thorough intraoral examination could not be carried out due to restricted mouth opening. Based on the chief complaint and clinical examination, a provisional diagnosis of left buccal space infection secondary to trauma was given. Screening radiographs, orthopantomogram, and Paranasal sinus (PNS) view, brought by the patient, were evaluated but were noncontributory, as no fractures were evident (Figure 1, 2).

Ultrasound scan was advised, which revealed soft tissue edema with streaks of hypoechoic fluid involving the deep subcutaneous plane of left cheek region, with no mention regarding the possible etiology of the same. The patient was advised admission for administration of IV antibiotics and surgical management, but patient refused, based on the same citing financial constraints and therefore he was discharged with advice on oral antibiotics. However, the patient reported back to us after 3 days with a dumb-bell shaped swelling on the left side of the face extending above the zygomatic arch involving superficial temporal space (Figure 3).

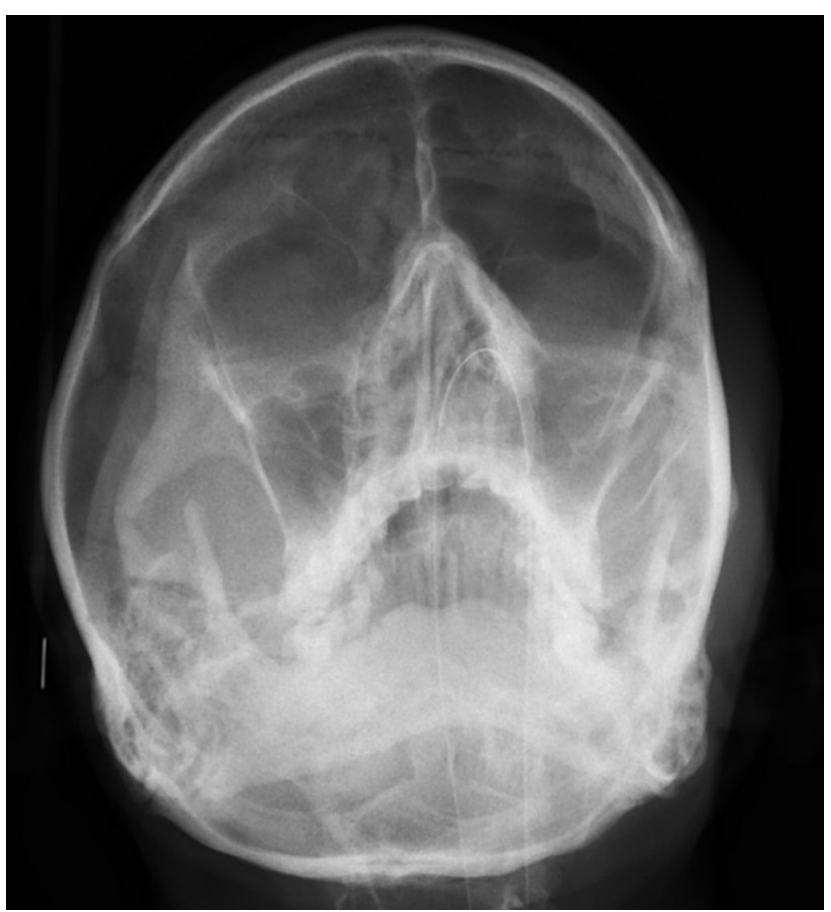

Figure 2. PNS x-ray view does not reveal any abnormality. 


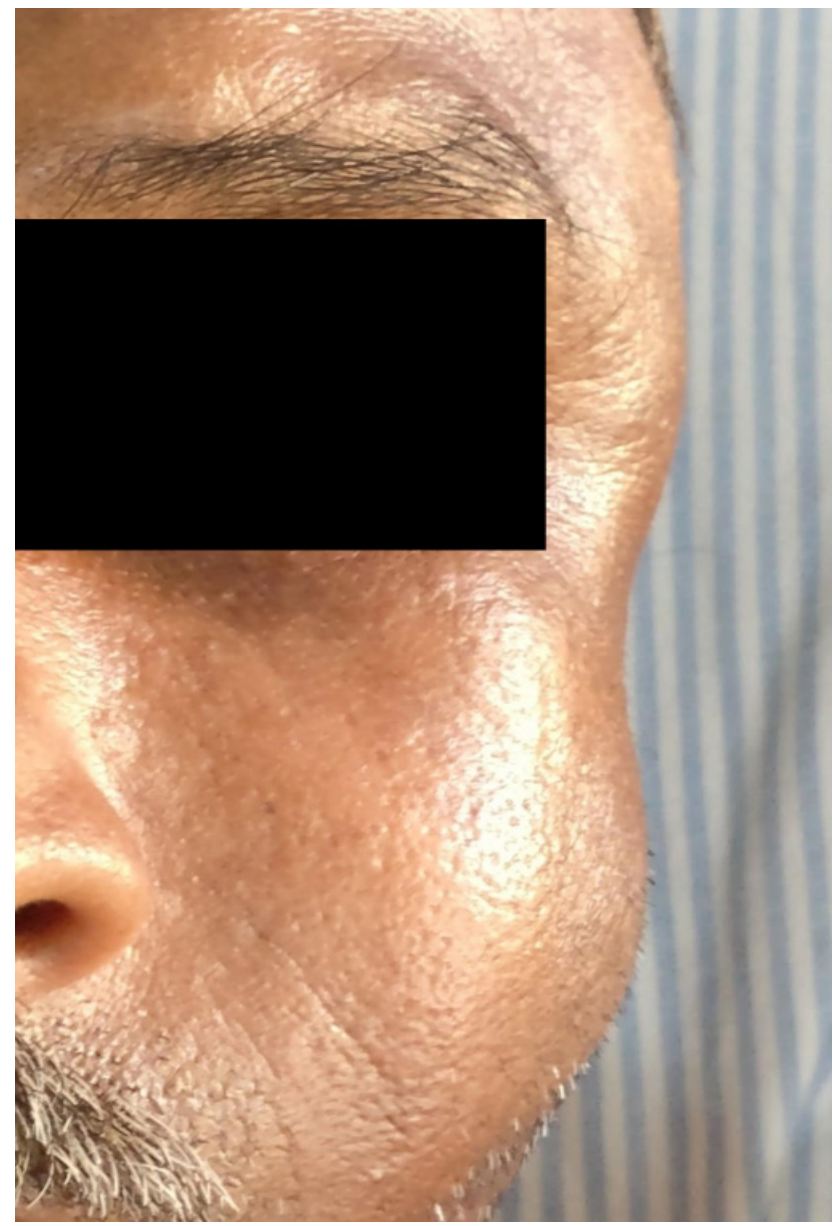

Figure 3. Classical dumb-bell shaped swelling of left side of face indicative of superficial temporalis space infection.

A contrast-based CT scan was done to define the extent of swelling, and also to look into the possible etiology of the same. Upon evaluation, ill-defined peripherally enhancing relatively hypodense collection with air foci was noted involving the left masticator space with infratemporal extension into the left buccal space (Figures 4, 5). These findings were suggestive of buccal space infection with extension into the superficial temporal space. No further insight was provided regarding the possible source of this non-resolving infection.
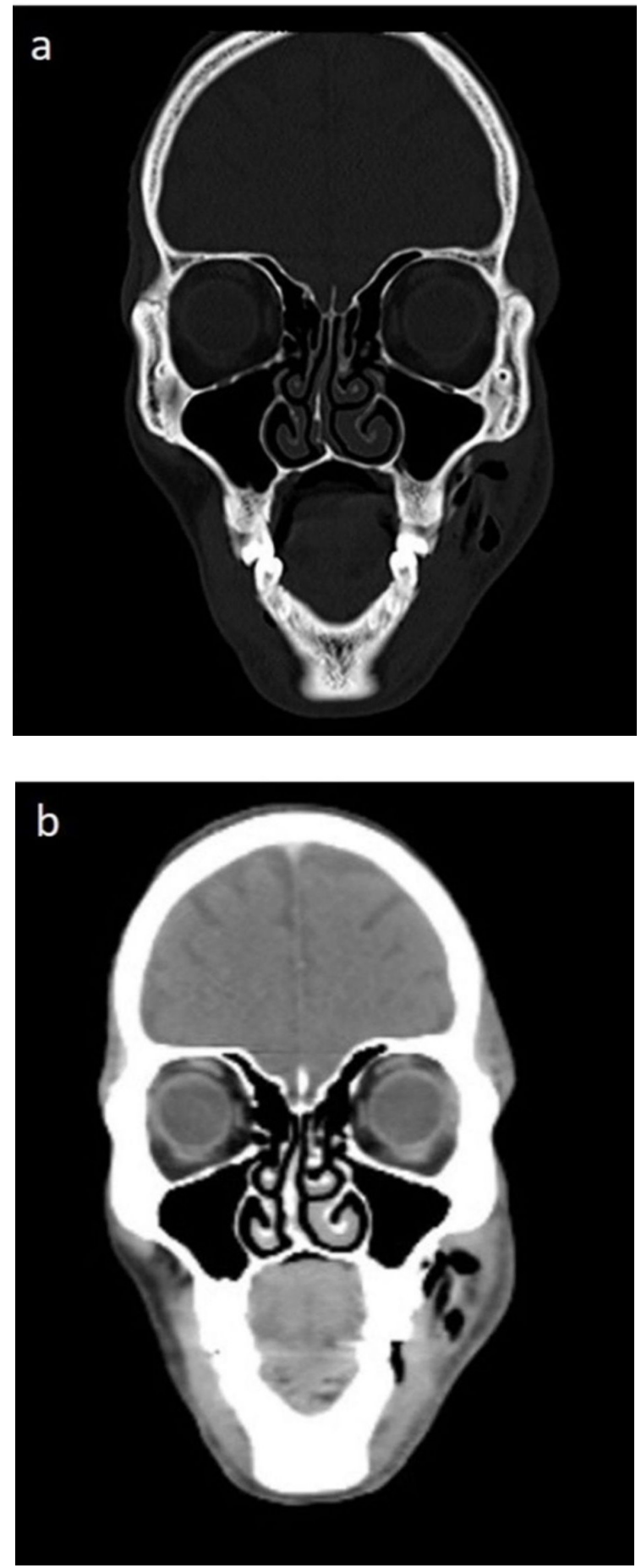

Figure 4. Coronal CT cuts in bone window (4a) and contrastbased (4b) shows ill-defined peripherally enhancing hypodense collection with air foci seen in left buccal space and with infratemporal extension. 

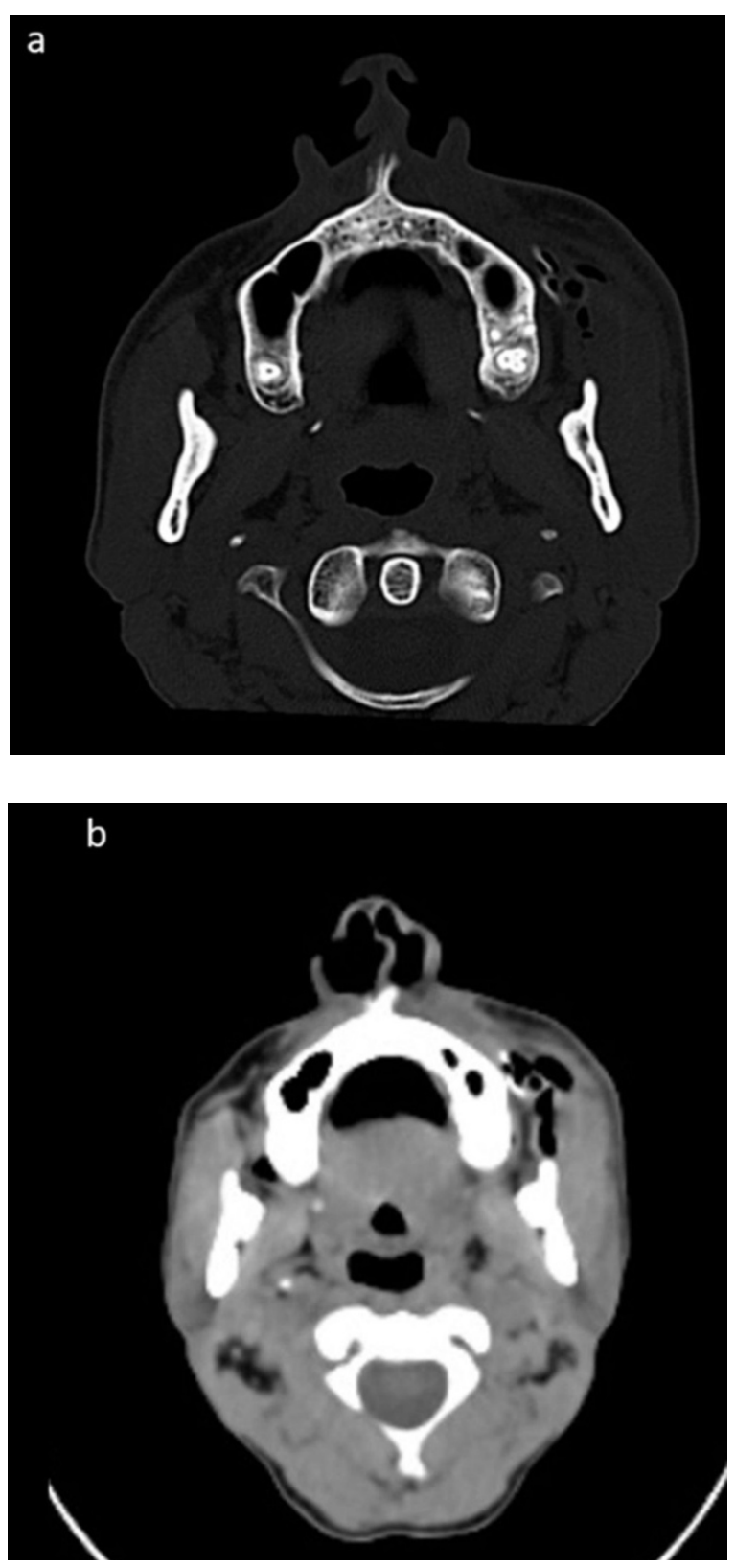

Figure 5. Bone window (5a) and Contrast-based (5b) CT axial section showing peripherally enhanced air pockets along with hypodense collection beneath the left buccinator muscle.

With the progression of the infection, patient agreed for admission and surgical exploration. Since there was no clear indication of a possible embedded foreign object from the patient's history, clinical examination, ultrasound scan or CT scan, an MRI scan was not considered. Patient was taken up for a thorough debridement and exploration of the affected area under general anesthesia. Upon forcefully opening the mouth, a hard palpable mass was felt along the posterior aspect of left buccal mucosa. The existing vestibular incision was extended to gain accessibility to the dubious mass. On further careful dissection into the buccal space, a $6 \mathrm{~cm}$ long wooden piece was retrieved (Figures 6,7).

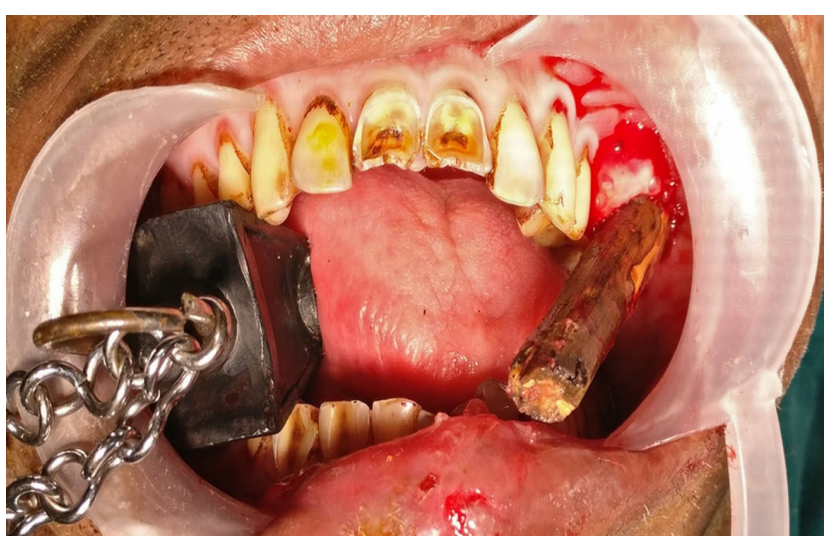

Figure 6. Intra-op picture showing the retrieval of wooden foreign object from left buccal space.

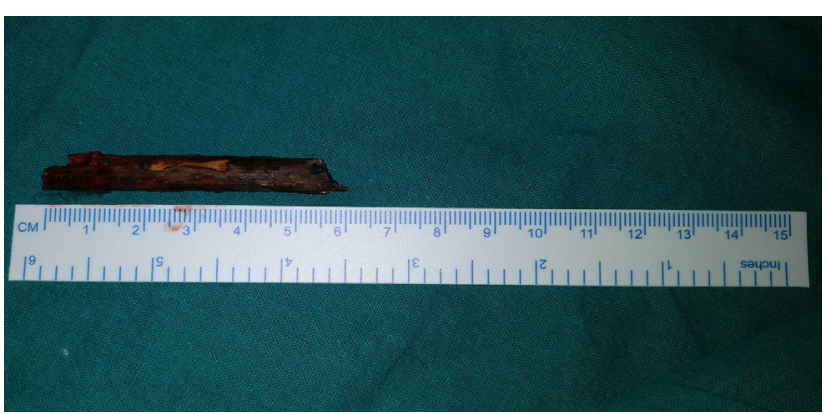

Figure 7. Retrieved wooden object measuring $6 \mathrm{~cm}$ in length.

The hemostat was extended superiorly in the buccal space to approach the superficial temporal space, and pus was drained and sent for culture and sensitivity testing. A dependent drainage was established and a long corrugated rubber drain was secured to the oral mucosa. The patient was continued on the triple antibiotic therapy (AmoxicillinClavulanate, Metronidazole and Gentamycin) while the culture-sensitivity report of the pus sample was awaited. Regular irrigation was done through the drain. Post 48 hours, culture report showed presence of Pseudomonas aeruginosa which was sensitive to Genatmycin. Hence, the patient was continued on the same antibiotics for another 5 days. The drain was removed 3 days post-surgery once there were no signs of active pus discharge and resolution of infection was seen. The closure of the intra-oral wound was done by a single suture.

The patient was discharged 7 days after surgery and was continued on oral antibiotics for 3 days and mouth opening exercises. On 1-week follow-up, the patient showed significant improvement in mouth opening and complete resolution of the infection (Figure 8). 


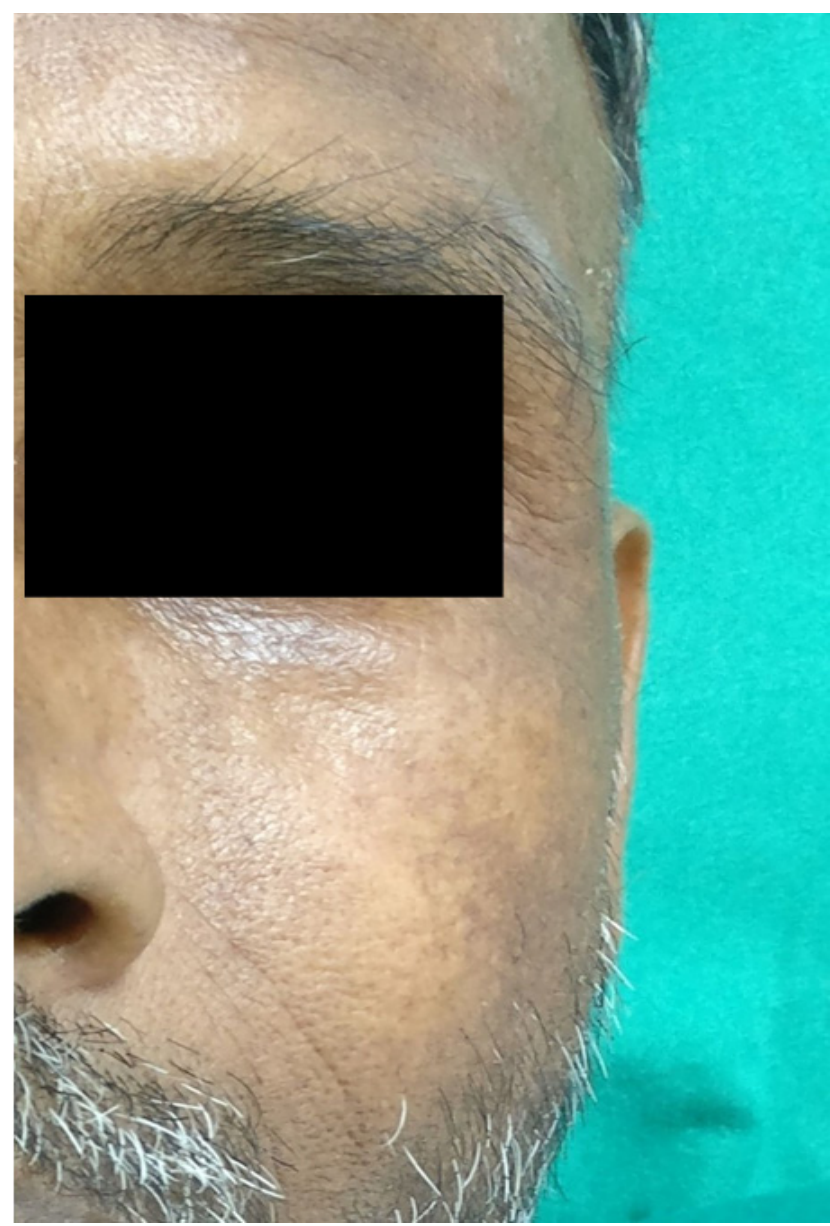

Figure 8. 2 weeks post-surgery showing complete resolution of the swelling.

\section{Discussion}

The correct diagnosis of an embedded foreign object in oral soft tissues can be challenging if proper history is not elicited. The term Jael syndrome is used when an impalement injury occurs in the craniofacial region [4]. As per de Santana Santos, a facial impalement injury in the oral cavity falls in the zone III, which lies between the angle of mandible and base of the skull [5]. Numerous materials have been reported in various penetrating wounds, but wood splinters and glass pieces are the most commonly encountered foreign body.

Wooden foreign object has been one of the frequently reported as a penetrating material in the facial region secondary to trauma. There have been numerous reports of long standing wooden foreign object in the facial region which later presented as sinus opening or pus discharge or persistent swelling or trismus [6-8]. Ahmed et al. reported a case series of three patients who presented with draining sinus in facial region which was attributed to embedded wooden foreign object [9].

In the case of a wooden object insertion, only $15 \%$ are detected on plain radiographs and are therefore often missed $[10,11]$. Further, a failure to diagnose or localize a wooden foreign body can lead to serious infectious complications as the wooden object can serve as an excellent source of various microorganisms. Because of its porous consistency along with organic nature, wood is an excellent medium for microorganisms and may result in an abscess, fistula formation, or space infection if the object is not removed in time [12].

CT imaging is considered as the best modality to visualize any deeply embedded wooden object which appears as air pockets or bubbles. However, with time, the wooden object imbibes water from the surrounding resulting in increased radiodensity and is mostly interpreted as soft tissue inflammation [12-14].

A superficially located wooden object can be identified by ultrasonography, which produces a detectable echogenic structure with acoustic shadowing on ultrasonography. However, this can be a diagnostic challenge if the object lies close to the bone or is located in deeper tissue planes [15]. In the present case, it was possible that the wooden foreign object was obscured by the zygomatic arch and hence, could not be identified by ultrasound scan. If the CT scan fails to reveal a wooden fragment, MRI has shown to be more sensitive in localizing a wooden object in the soft tissue. T-1 weighted image provides maximum contrast between wood and fat [2].

In an in vitro study by Javadrashid et al., all embedded foreign objects were best visualized by a CT scan, except for a wooden object. A wooden object measuring more than $0.5 \mathrm{~mm}$ was best detected by ultrasound scan. MRI was found to be the least useful imaging technique for visualizing a foreign object in maxillofacial region [16]. In another similar in vitro study by Shokri et al., a comparative analysis was done between Cone-beam CT (CBCT), MRI and ultrasound for their sensitivity and specificity in detecting foreign object in facial region. Their study revealed that a CBCT was most sensitive in detecting a glass particle or a pebble. However, none of these imaging modalities could clearly visualize a wooden foreign object. Also, they suggested that MRI should not be used as $1^{\text {st }}$ choice of imaging modality, especially if the nature of foreign object is not known, because of significant artifacts and high cost [17].

Kaviani et al. compared the efficacy of CBCT scan with conventional $\mathrm{CT}$ scan in localizing a foreign object in facial region. They found that CBCT scan with NewTom and Planmeca were more effective in visualization of foreign object as compared to a conventional CT scan [18]. Recently, software like Mimics (Materialise Interactive Medical Image Control Systems) have been used with CBCT images which has shown to be more effective in determining the location and type of foreign object. However, this study had evaluated for the presence of endodontic file in the maxillofacial region and the 
application for the same in detecting wooden foreign object should be further investigated [19]. There has been a report of utilization of navigation system guided by a dental splint to localize and remove a foreign object from the region of maxillary sinus in a minimally invasive manner. However, cost and the availability of such systems are the main limiting factors [20].

The buccal space is the anatomical area found in between the buccinators muscle medially and the muscles of facial expressions and skin laterally. The contents of the buccal space include buccal fat pad, parotid duct, facial arteries and vein, buccal arteries and lymphatic channels. Buccal fat pad occupies the majority of the buccal space and is formed of 4 extensions, namely - medial, lateral, anterior and superficial extensions. The buccal space communicates with the surrounding masticator spaces. The superficial temporal space communication with buccal space is established by the temporal extension of buccal fat pad [21]. Presentation of a space infection in the form of swelling or trismus in a trauma patient, in the absence of any fractures should raise a strong suspicion for deeply embedded foreign objects. In the present case, inability to carry out thorough intra-oral examination due to restricted mouth opening and worsening of infection mandated the patient to be taken up for surgical exploration under general anesthesia. The mouth opening restriction was attributed to the involvement of masticator space, and there was a significant improvement in mouth opening with the resolution of the infection. The presentation of classic dumb-bell shaped appearance was indicative of temporal space infection, which has the potential to cause some fatal complications.

The delayed presentation to our clinic, and also the unclear history, compounded the diagnostic dilemma, which shows the importance of thorough history elicitation and clinical examination and correlation of the same with the diagnostic imaging in discussion with the oral radiologist. Based on our literature review, it is advisable to use the ultrasound scan and CBCT scan, if available, for investigating a possible embedded foreign object in the maxillofacial region.

\section{Conclusion}

Any patient presenting with an unclear history about the mechanism of injury should always be evaluated for penetrating wounds, even in the absence of extra-oral wound, which would guide towards further investigation and management. Detection of the foreign object cannot be solely relied on imaging modalities and should be correlated with the patient history and proper clinical examination.

\section{References}

1. Mohan S, Varghese G, Kumar S, Subramanian DP. Penetrating facial injury by a wooden $\log$. Natl J Maxillofac
Surg. 2014;5:228-231.

2. Krimmel M, Cornelius CP, Stojadinovic S, Hoffmann $\mathrm{J}$, Reinert S. Wooden foreign bodies in facial injury: a radiological pitfall. Int J Oral Maxillofac Surg. 2001;30:445447.

3. Loganathan K, Chacko JP, Saravanan BS, Vaithilingam B. Management of wooden foreign bodies in craniofacial region. J Oral Biol Craniofac Res. 2012;2:210-212.

4. Klein B, McGahan JP. Thorn synovitis: CT diagnosis. J Comput Assist Tomogr. 1985;9:1135-1136.

5. de Santana Santos T, Avelar RL, Melo AR, de Moraes HH, Dourado E. Current approach in the management of patients with foreign bodies in the maxillofacial region. J Oral Maxillofac Surg. 2011;69:2376-2382.

6. Auluck A, Behanan AG, Pai KM, Shetty C. Recurrent sinus of the cheek due to a retained foreign body: report of an unusual case. Br Dent J. 2005;198:337-339.

7. Vikram A, Mowar A, Kumar S. Wooden foreign body in the zygomatic region for 2 years. J. Maxillofac Oral Surg. 2012;11:96-100.

8. Joyce S, Rao Sripathi BH, Mampilly MO, Firdoose Nyer CS. Foreign body granuloma. J Maxillofac Oral Surg. 2014;13:351-354.

9. Ahmed SS, Abid S, Al-Thobaiti Y, Baliga SD, Fayyaz M. Longstanding Unrecognized Wooden Foreign Bodies in Oro-facial Region, Report of three Cases. American Journal of Public Health. 2015;3:157-161.

10. Anderson MA, Newmeyer WL 3rd, Kilgore ES Jr. Diagnosis and treatment of retained foreign bodies in the hand. Am J Surg. 1982;144:63-67.

11. Robinson PD, Rajayogeswaran V, Orr R. Unlikely foreign bodies in unusual facial sites. Br J Oral Maxillofac Surg. 1997;35:36-39.

12. Dalley RW. Intraorbital wood foreign bodies on CT: use of wide bone window settings to distinguish wood from air. AJR Am J Roentgenol. 1995;164:434-435.

13. Dort JC, Robertson D. Nonmetallic foreign bodies of the skull base: a diagnostic challenge. J Otolaryngol. 1995 Feb;24(1):69-72.

14. Specht CS, Varga JH, Jalali MM, Edelstein JP. Orbitocranial wooden foreign body diagnosed by magnetic resonance imaging. Dry wood can be isodense with air and orbital fat by computed tomography. Surv Ophthalmol. 1992;36:341344.

15. Aras MH, Miloglu O, Barutcugil C, Kantarci M, Ozcan E, Harorli A. Comparison of the sensitivity for detecting foreign bodies among conventional plain radiography, computed tomography and ultrasonography. Dentomaxillofac Radiol. 2010;39:72-78.

16. Javadrashid R, Fouladi DF, Golamian M, Hajalioghli P, Daghighi MH, Shahmorady Z, et al. Visibility of different foreign bodies in the maxillofacial region using plain radiography, CT, MRI and ultrasonography: an in vitro study. Dentomaxillofac Radiol. 2015;44:20140229.

17. Shokri A, Jamalpour M, Jafariyeh B, Poorolajal J, Sabet NK. Comparison of Ultrasonography, Magnetic Resonance Imaging and Cone Beam Computed Tomography for 
Detection of Foreign Bodies in Maxillofacial Region. J Clin Diagn Res. 2017;11:TC15-TC19.

18. Kaviani F, Javad Rashid R, Shahmoradi Z, Gholamian M. Detection of foreign bodies by spiral computed tomography and cone beam computed tomography in maxillofacial regions. J Dent Res Dent Clin Dent Prospects. 2014;8:166-171.

19. Asif MK, Nambiar P, Khan IM, Aziz ZABCA, Noor NSBM, Shanmuhasuntharam P, et al. Enhancing the threedimensional visualization of a foreign object using Mimics software. Radiol Case Rep. 2019;14:1545-1549.

20. Sukegawa S, Yoneda S, Kanno T, Tohmori H, Furuki Y. Optical surgical navigation-assisted removal of a foreign body using a splint to simplify the registration process: a case report. J Med Case Rep. 2019;13:209.

21. Kim HC, Han MH, Moon MH, Kim JH, Kim IO, Chang KH. CT and MR imaging of the buccal space: normal anatomy and abnormalities. Korean J Radiol. 2005;6:22-30. 